\title{
THE EULER-LAGRANGE EXPRESSION AND DEGENERATE LAGRANGE DENSITIES
}

\author{
D. LOVELOCK*
}

(Received 12 May 1970, revised 19 August 1970)

Communicated by E. Strzelecki

\section{Introduction and motivation}

It is well known that many of the field equations from theoretical physics (e.g. Einstein field equations, Maxwell's equations, Klein-Gordon equation) can be obtained from a variational principle with a suitably chosen Lagrange density. In the case of the Einstein equations the corresponding Lagrangian is degenerate (i.e., the associated Euler-Lagrange equations are of second order whereas in general these would be of fourth order), while in the cases of the Maxwell and Klein-Gordon equations the Lagrangian usually used is not degenerate. However, it is not generally realized that there exist degenerate Lagrange densities which also give rise to these last two field equations. In this note the general structure of this type of degenerate Lagrange density is examined.

We shall concentrate our attention on $m$ quantities $\rho^{A}(A=1, \ldots, m)$ which in general are each functions of position i.e.

$$
\rho^{A}=\rho^{A}\left(x^{j}\right) .
$$

Under transformations of the type

$$
\bar{x}^{a}=\bar{x}^{a}\left(x^{j}\right)
$$

we shall assume that the $\rho^{A}$ transform according to the law ${ }^{1}$

$$
\vec{\rho}^{A}=C_{B}^{A} \rho^{B},
$$

where the $C_{B}^{A}$ are functions of $\bar{x}^{a}$ (or $x^{j}$ ) and are completely determined by the transformation (1.1). To fix ideas we cite four examples all of which fall into the above category.

* This work was supported by a grant from the National Research Council of Canada.

1 Unless otherwise noted the summation convention will apply, whereby repeated capital indices $A, B, \ldots$ will be summed from 1 to $m$ and repeated latin indices $a, b, \ldots, i, j, \ldots$ from 1 to $n$. 
(i) Scalar field $\phi$. If $\phi$ is a scalar field the counterpart of (1.2) reads

$$
\phi=\phi,
$$

in which case $m=1, \rho^{A}=\phi$ and $C_{B}^{A}=1$.

(ii) Vector field $\psi_{i}$. In this case $m=n, \rho^{A}=\psi_{i}$ and $C_{B}^{A}=B_{a}^{i}\left(=\partial x^{i} / \partial \bar{x}^{a}\right)$ since a vector field transforms according to the law ${ }^{1}$

$$
\bar{\psi}_{a}=B_{a}^{i} \psi_{i}
$$

(iii) Tensor field $g_{i j}$. Here $m=n^{2}, \rho^{A}=g_{i j}$ and $C_{B}^{A}=B_{a}^{i} B_{b}^{j}$ corresponding to

$$
\bar{g}_{a b}=B_{a}^{i} B_{b}^{j} g_{i j} .
$$

(iv) Non-tensorial field $\alpha_{i j}$. If $\alpha_{i j}$ are $n^{2}$ quantities which transform according to ${ }^{2}$

$$
\bar{\alpha}_{a b}=\sum_{i=1}^{n} \sum_{j=1}^{n}\left[\sum_{c=1}^{n} \sum_{d=1}^{n} B_{a c}^{i} B_{b d}^{j} A_{i}^{c} A_{j}^{d}+B_{a}^{i} B_{b}^{j}\right] \alpha_{i j}
$$

where we have put

$$
B_{a c}^{i}=\partial B_{a}^{i} / \partial \bar{x}^{c} \text { and } A_{i}^{c}=\partial \bar{x}^{c} / \partial x^{i},
$$

then under these circumstances

$$
C_{B}^{A}=\sum_{c=1}^{n} \sum_{d=1}^{n} B_{a c}^{i} B_{b d}^{j} A_{i}^{c} A_{j}^{d}+B_{a}^{i} B_{b}^{j} .
$$

From these examples it is evident that $\rho^{A}$ may represent, on the one hand, the components of an arbitrary relative tensor field and, on the other hand, certain quantities which are manifestly non-tensorial in character.

We now assume that we are given a quantity $L$-the Lagrangian. It is furthermore supposed that $L$ is a function of $\rho^{A}$ and its first $M$ partial derivatives together with $q$ arbitrary preassigned functions of position $\lambda^{\alpha}(\alpha=1, \ldots, q)$ and the first $Q$ partial derivatives of $\lambda^{\alpha}$, i.e.

$$
L=L\left(\rho^{A} ; \rho^{A},{ }_{i_{1}} ; \ldots ; \rho^{A}{ }_{i_{1} \cdots i_{M}} ; \lambda^{\alpha} ; \lambda^{\alpha},{ }_{i_{1}} ; \ldots ; \lambda^{\alpha},{ }_{i_{1} \ldots i_{Q}}\right),
$$

where a comma denotes partial differentiation.

With $L$ we can always associate the Euler-Lagrange expression $E_{A}(L)$ defined by

$$
E_{A}(L)=\frac{\partial L}{\partial \rho^{A}}+\sum_{r=1}^{M}(-1)^{r} \frac{\partial^{r}}{\partial x^{i_{1}} \ldots \partial x^{i_{r}}}\left(\frac{\partial L}{\partial \rho^{A}{ }_{, i_{1} \ldots i_{r}}}\right),
$$

where, as mentioned above, the $\lambda^{\alpha}$ are not varied but are assumed to be preas-

2 The summation convention does not apply to this example. 
signed functions of position. In general the Euler-Lagrange expression (1.4) will be of order $2 M$ in $\rho^{A}$ (and order $M+Q$ in $\lambda^{\alpha}$ ). If the order of this expression in $\rho^{A}$ is less than $2 M$ the corresponding Lagrangian is called degenerate. For examples of this see [2], [3] and [4].

In order to ensure that the so-called action integral corresponding to (1.3) viz.

$$
\int \ldots \int L d x^{1} \ldots d x^{n}
$$

is an invariant, we assume that $L$ is a scalar density, i.e. under (1.1)

$$
\bar{L}=B L
$$

where

$$
B=\operatorname{det}\left|B_{a}^{i}\right| .
$$

In theoretical physics the role played by the Euler-Lagrange equations

$$
E_{A}(L)=0
$$

is well known. It is usually possible to derive the field equations of physics from a variational principle with a suitably chosen Lagrangian $L$. To illustrate this we briefly discuss three important cases.

(a) Symmetric tensor field: Einstein vacuum field equations.

Consider the Lagrangian $L$ given by

$$
L\left(g_{i j} ; g_{i j, k} ; g_{i j, k h}\right)=\sqrt{g} g^{i j} R_{i j h}^{h},
$$

where $g=\operatorname{det}\left|g_{i j}\right|, g^{i j}$ are characterised by

$$
g^{i j} g_{k j}=\delta_{k}^{i}
$$

and

with

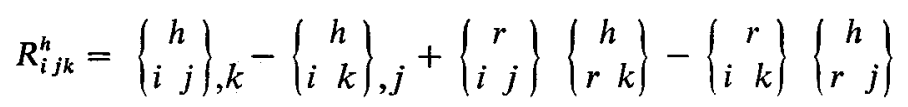

$$
\left\{\begin{array}{c}
h \\
i \quad j
\end{array}\right\}=\frac{1}{2} g^{h k}\left(g_{i k, j}+g_{j k, i}-g_{i j, k}\right) .
$$

With the correspondence

$$
\rho^{A}=g_{i j}, \lambda^{\alpha}=0,
$$

in (1.3) the associated Euler-Lagrange expression (1.4) is ${ }^{3}$

$$
E^{i j}(L)=-\sqrt{g}\left(g^{i h} g^{j k} R_{h k l}^{l}-\frac{1}{2} g^{i j} g^{h k} R_{h k l}^{l}\right)
$$

${ }^{3}$ See e.g. [5] p. 258. 
and the Euler-Lagrange equations (1.6) are just the Einstein field equations in vacuo.

(b) Scalar field: Klein-Gordon Equation.

If we consider the Lagrangian

$$
L\left(\phi ; \phi_{, i} ; g_{i j}\right)=\frac{1}{2} \sqrt{g}\left(g^{i j} \phi_{, i} \phi_{, j}+k^{2} \phi^{2}\right),
$$

(where $k=$ constant), and use the correspondence

$$
\rho^{A}=\phi, \lambda^{\alpha}=g_{i j},
$$

then (1.4) becomes

$$
E(L)=\sqrt{g}\left(g^{i j} \phi_{\mid i j}-k^{2} \phi\right),
$$

where the vertical bar denotes covariant differentiation with respect to $g_{i j}$. The Euler-Lagrange equation in this case is the Klein-Gordon equation.

(c) Vector field: Maxwell's equation

By choosing

$$
L\left(\psi_{1} ; \psi_{i, j} ; g_{i j}\right)=\frac{1}{2} \sqrt{g} F_{i j} F_{h k} g^{i h} g^{j k}
$$

where

$$
F_{i j}=\psi_{i, j}-\psi_{j, i}
$$

and by identifying

$$
\rho^{A}=\psi_{i}, \lambda^{\alpha}=g_{i j},
$$

we find that (1.4) reads

$$
E^{i}(L)=2 \sqrt{g} g^{i h} g^{j k} F_{h k \mid j} .
$$

The corresponding Euler-Lagrange equations are Maxwell's equations in the absence of sources.

Of these three Lagrangians i.e. (1.7), (1.9) and (1.11), only one is degenerate viz. (1.7). However, it is not generally realized that in the other two cases it is possible to choose Lagrange densities which are degenerate but which still yield (1.10) and (1.12) as the corresponding Euler-Lagrange expressions. These Lagrangians are

$$
L\left(\phi ; \phi_{, i} ; \phi_{, i j} ; g_{i j} ; g_{i j, k}\right)=\frac{1}{2} \sqrt{g} \phi\left(g^{i j} \phi_{\mid i j}-k^{2} \phi\right),
$$

and

$$
L\left(\psi_{i} ; \psi_{i, j} ; \psi_{i, j k} ; g_{i j} ; g_{i j, k}\right)=\sqrt{g} \psi_{i} g^{i h} g^{j k} F_{h k \mid j},
$$

respectively. Aside from their degeneracy, the Lagrangians (1.7), (1.13) and (1.14) have something else in common - they all have the same structure viz. 


$$
a \rho^{A} E_{A}(L)
$$

where $a$ is a constant, in the general case. This raises three obvious questions:

1. If $L$ is a scalar density and $\rho^{A}$ transform according to (1.2) is $\rho^{A} E_{A}(L)$ a scalar density?

2. If $\rho^{A} E_{A}(L)$ is a scalar density and we regard it as a new Lagrangian, is it always degenerate?

3. If $\rho^{A} E_{A}(L)$ is a degenerate Lagrange density are its Euler-Lagrange equations always $E_{A}(L)=0$, up to a constant?

These three questions will be answered in the next sections by means of Theorems 2, 4 and 6 respectively.

\section{Certain properties of the Euler-Lagrange expression}

The main purpose of this section is to establish the transformation law for $E_{A}(L)$ under (1.1) where it is assumed that $\rho^{A}$ and $L$ transform according to (1.2) and (1.5) respectively.

To this end we introduce the following notation. If $F_{\ldots}^{\ldots}$ is any function of $x^{i}$ then

and

$$
F_{\cdots, i_{0}}^{\cdots}=F_{\ldots}^{\cdots}
$$

$$
F_{\ldots, i_{0} i_{1} \ldots i_{r}}^{\cdots}=\partial F_{\ldots, i_{0} i_{1} \ldots i_{r-1}} / \partial x^{i_{r}}
$$

for $r=1,2, \ldots$. Similarly if $G_{\ldots}^{\ldots}$ is any function of $\bar{x}^{a}$ then

and

$$
G_{\ldots, a_{0}}^{\ldots}=G_{\ldots}^{\cdots}
$$

$$
G_{\ldots, a_{0} a_{1} \ldots a_{r}}=\partial G_{\ldots, a_{0} a_{1} \ldots a_{r-1}} / \partial \bar{x}^{a_{r}}
$$

for $r=1,2, \ldots$. In view of this the summation convention will not apply to the indices $i_{0}$ or $a_{0}$.

It is possible to rewrite (1.4) in a slightly more concise form by introducing the following definitions:

$$
\begin{gathered}
L_{A}^{i_{0}}=\partial L / \partial \rho^{A}, i_{0}\left(=\partial L / \partial \rho^{A}\right), \\
L_{A}^{i_{0} i_{1} \ldots i_{r}}=\partial L / \partial \rho^{A},{ }_{i_{0} i_{1} \ldots i_{r}}
\end{gathered}
$$

for $1 \leqq r \leqq M$. In this case (1.4) becomes

$$
E_{A}(L)=\sum_{r=0}^{M}(-1)^{r} L_{A}^{i_{0} i_{1} \ldots i_{r}}{ }_{i_{0} i_{1} \ldots i_{r}} .
$$

In order to obtain the transformation law for $E_{A}(L)$ we will require the 
transformation properties of $\bar{\rho}^{A}{ }_{, a_{0} a_{1} \ldots a_{k}}$ for all $k, 0 \leqq k \leqq M$. We shall obtain these properties in the form of a recurrence relation. In view of (1.2) it is clear that $\bar{\rho}^{A}{ }_{, a_{0} a_{1} \ldots a_{k}}$ will be linear in $\rho^{B}{ }_{, i_{0} i_{1} \ldots i_{j}}$ for all $j, 0 \leqq j \leqq k$, and can thus be expressed in the form

$$
\vec{\rho}_{, a_{0} a_{1} \ldots a_{k}}^{A}=\sum_{j=0}^{k} \alpha_{B a_{0} a_{1} \ldots a_{k}}^{A i_{0} i_{1} \ldots i_{j}} \rho_{, i_{0} i_{1} \ldots i_{j}}^{B},
$$

where $\alpha_{B a_{0} a_{1} \ldots a_{k}}^{A i_{i} i_{1} \ldots i_{j}}$ are functions of $\bar{x}^{a}$. Differentiation of (2.1) with respect to $\bar{x}^{a_{k+1}}$ yields

$$
\begin{gathered}
\bar{\rho}_{, a_{0} a_{1} \ldots a_{k+1}}^{A}=\sum_{j=1}^{k+1}\left[\alpha_{B a_{0} a_{1} \ldots a_{k}}^{A i_{0} i_{1} \ldots i_{j}-1} B_{a_{k+1}}^{i_{j}}+\alpha_{B a_{0} a_{1} \ldots a_{k}, a_{k+1}}^{A i_{0} i_{1} \ldots i_{j}}\right] \rho_{, i_{0} i_{1} \ldots i_{j}}^{B}+ \\
+\alpha_{B a_{0} a_{1} \ldots a_{k}, a_{k+1}}^{A} \rho^{B}
\end{gathered}
$$

with the understanding that

$$
\alpha_{B a_{0} a_{1} \ldots a_{k}}^{A i_{0} i_{1} \ldots i_{k+1}}=0
$$

A comparison of (2.2) with (2.1) [with $k$ replaced by $(k+1)$ in the latter] yields the following. If $k$ is any integer, $0 \leqq k+1 \leqq M$,

$$
\alpha_{B a_{0} a_{1} \ldots a_{k+1}}^{A i_{0} i_{1} \ldots i_{j}}= \begin{cases}\alpha_{B a_{0} a_{1} \ldots a_{k}, a_{k+1}}^{A i_{0}} & \text { if } j=0 \text { and } k \geqq 0, \\ \alpha_{B a_{0} a_{1} \ldots a_{k}}^{A i_{0} i_{1} \ldots i_{j-1}} B_{a_{k+1}}^{i_{j}}+\alpha_{B a_{0} a_{1} \ldots a_{k}, a_{k+1}}^{A i_{0} i_{1} \ldots i_{j}} & \text { if } 1 \leqq j \leqq k+1, \\ 0 & \text { if } j>k+1\end{cases}
$$

Obviously

$$
\alpha_{B a_{0}}^{A i_{0}}=C_{B}^{A} .
$$

By means of (2.3) we can prove the

LEMMA. If under (1.1) $\rho^{A}$ and L transform according to (1.2) and (1.5) respectively then for $1 \leqq k \leqq M-1$

$$
\begin{aligned}
& \sum_{p=0}^{k-1}(-1)^{p} L_{B}{ }^{i_{0} i_{1} \ldots i_{M-p}}{ }_{, i_{M-p}} i_{M-p-1} \ldots i_{M-k+1}+(-1)^{k} L_{B}{ }^{i_{0} i_{1} \ldots i_{M-k}}= \\
& =\sum_{j=0}^{k-1}(-1)^{j}\left\{\sum_{p=0}^{k-j-1}(-1)^{p} \bar{L}_{A}^{a_{0} a_{1} \ldots a_{M-p}}, a_{M-p} a_{M-p-1 \ldots a_{M-k+j+1}}+\right. \\
& \left.+(-1)^{k-j} \bar{L}_{A}^{a_{0} a_{1} \ldots a_{M-k+j}}\right\} \alpha_{B a_{0} a_{1} \ldots a_{M-k+j-1}}^{A i_{0} i_{1} \ldots i_{M-k-1}} B_{a_{M-k+j}}^{i_{M-k}} / B+ \\
& +(-1)^{k} \bar{L}_{A}^{a_{0} a_{1} \ldots a_{M}} \alpha_{B a_{0} a_{1} \ldots a_{M}-1}^{A i_{0} i_{1} \ldots i_{M}-k-1} B_{a_{M}}^{i_{M}-k} / B .
\end{aligned}
$$


Proof. We shall prove this by induction over $k$. With $k=1(2.5)$ reads

$$
\begin{aligned}
& L_{B}^{i_{0} i_{1} \ldots i_{M}}{ }_{, i_{M}}-L_{B}^{i_{0} i_{1} \ldots i_{M-1}}=\left(\bar{L}_{A}^{a_{0} a_{1} \ldots a_{M}}{ }_{, a_{M}}-L_{A}^{a_{0} a_{1} \ldots a_{M-1}}\right) \times \\
& \times \alpha_{B a_{0} a_{1} \ldots a_{M-2}}^{A i_{0} i_{1} \ldots i_{M-2}} B_{a_{M-1}}^{i_{M-1}} / B-L_{A}^{a_{0} a_{1} \ldots a_{M}} \alpha_{B_{10} a_{1} \ldots a_{M-1}}^{A a_{0} i_{1} \ldots i_{M-2}} B_{a_{M}}^{i_{M-1}} / B .
\end{aligned}
$$

From (1.5) and (2.1) we find

$$
L_{B}^{i_{0} i_{1} \ldots i_{M}}=\bar{L}_{A}^{a_{0} a_{1} \ldots a_{M}} \alpha_{B a_{0} a_{1} \ldots a_{M}}^{A i_{i} i_{1} \ldots i_{M}} / B,
$$

which, by virtue of (2.3), can be written as

$$
L_{B}^{i_{0} i_{1} \ldots i_{M}}=\widetilde{L}_{A}^{a_{0} a_{1} \ldots a_{M}} \alpha_{B a_{0} a_{1} \ldots a_{M-1}}^{A i_{0} i_{1} \ldots i_{M}-1} B_{a_{M}}^{i_{M}} / B .
$$

In view of the fact that

$$
\frac{\partial}{\partial x^{i}}\left(B_{a}^{i} / B\right)=0
$$

we thus have

(2.7) $L_{B}^{i_{0} i_{1} \ldots i_{M}}{ }_{, i_{M}}=\left(\bar{L}_{A}^{a_{0} a_{1} \ldots a_{M}}{ }_{, a_{M}} \alpha_{B a_{0} a_{1} \ldots a_{M-1}}^{A i_{0} i_{1} \ldots i_{M-1}}+\bar{L}_{A}^{a_{0} a_{1} \ldots a_{M}} \alpha_{B a_{0} a_{1} \ldots a_{M-1}, a_{M}}^{A i_{0} i_{1} \ldots i_{M-1}}\right) / B$.

From (1.5) and (2.1) we also see that

$$
L_{B}^{i_{0} i_{1} \ldots i_{M-1}}=\left(\bar{L}_{A}^{a_{0} a_{1} \ldots a_{M}} \alpha_{B a_{0} a_{1} \ldots a_{M}}^{A i_{0} i_{1} \ldots i_{M-1}}+\bar{L}_{A}^{a_{0} a_{1} \ldots a_{M-1}} \alpha_{B a_{0} a_{1} \ldots a_{M-1}}^{A i_{0} i_{1} \ldots i_{M-1}}\right) / B
$$

Subtraction of (2.8) from (2.7) yields

$$
\begin{aligned}
L_{B}^{i_{0} i_{1} \ldots i_{M}}{ }_{, i_{M}} & -L_{B}^{i_{0} i_{1} \ldots i_{M-1}}=\left(\bar{L}_{A}^{a_{0} a_{1} \ldots a_{M}}{ }_{, a_{M}}-\bar{L}_{A}^{a_{0} a_{1} \ldots a_{M-1}}\right) \alpha_{B a_{0} a_{1} \ldots a_{M-1}}^{A i_{i} i_{1} \ldots i_{M-1}} / B+ \\
& +L_{A}^{a_{0} a_{1} \ldots a_{M}}\left(\alpha_{B a_{0} a_{1} \ldots a_{M-1}, a_{M}}^{A i_{0} i_{1} \ldots i_{M-1}}-\alpha_{B a_{0} a_{1} \ldots a_{M}}^{A i_{0} i_{1} \ldots i_{M-1}}\right) / B,
\end{aligned}
$$

which is (2.6) when account is taken of (2.3). Hence (2.5) is valid for $k=1$.

We now assume (2.5) to be true for fixed $k$ and establish the validity of (2.5) with $k$ replaced by $k+1$, i.e. we wish to show that (2.5) implies

$$
\begin{aligned}
& \sum_{p=0}^{k}(-1)^{p} L_{B}^{i_{0} i_{1} \ldots i_{M-p}{ }_{.} i_{M-p} i_{M-p-1} \ldots i_{M-k+1} i_{M-k}}+(-1)^{k+1} L_{B}^{i_{i} i_{1} \ldots i_{M-k-1}}=
\end{aligned}
$$

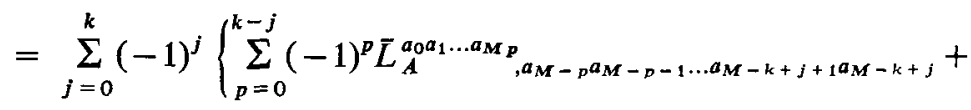

$$
\begin{aligned}
& \left.+(-1)^{k-j+1} \bar{L}_{A}^{a_{0} a_{1} \ldots a_{M-k+j-1}}\right\} \alpha_{B a_{0} a_{1} \ldots a_{M-k-2+j}}^{A i_{0} i_{1} \ldots i_{M-k-2}} B_{a_{M-k+j-1}}^{i_{M-k-1}} / B+ \\
& +(-1)^{k+1} \bar{L}_{A}^{a_{0} a_{1} \ldots a_{M}} \alpha_{B a_{0} a_{1} \ldots a_{M-1}}^{A i_{0} i_{1} \ldots i_{M-k-2}} B_{a_{M}}^{i_{M-k-1}} / B \text {. }
\end{aligned}
$$

We see that the first term on the left hand side of (2.9) is the derivative of the left hand side of (2.5) with respect to $x^{i_{M-k}}$. Consequently the left hand side of (2.9) can be expressed as the sum of six expressions, viz. 


$$
\begin{aligned}
& \sum_{j=0}^{k-1}(-1)^{j} \sum_{p=0}^{k-j}(-1)^{p} \bar{L}_{A}^{a_{0} a_{1} \ldots a_{M-n}}{ }_{, a_{M}-p} a_{M} \quad{ }_{n-1} \ldots a_{M-k+j+1} a_{M-k+j} \alpha_{B a_{0} a_{1} \ldots a_{M}-k+j-1}^{A i_{0} i_{1} \ldots i_{M-k-1}} / B+ \\
& +\sum_{j=0}^{k-1}(-1)^{j} \sum_{p=0}^{k-j-1}(-1)^{p} \bar{L}_{A}^{a_{0} a_{1} \ldots a_{M}-\eta}{ }_{, a_{M}-p \ldots a_{M-k+j+1}} \alpha_{B a_{0} a_{1} \ldots a_{M-k+j-1}, a_{M-k+j}}^{A i_{0} i_{1} \ldots i_{M}-k-1} / B+ \\
& +\sum_{j=0}^{k-1}(-1)^{k} \bar{L}_{A}^{a_{0} a_{1} \ldots a_{M-k+j}} \alpha_{B a_{0} a_{1} \ldots a_{M-k+j-1} i_{a_{M-k+j}} i_{1} \ldots i_{M-k-1}}^{j} / B+ \\
& +(-1)^{k} \bar{L}_{A}^{a_{0} a_{1} \ldots a_{M}}{ }_{, a_{M}} \alpha_{B a_{0} a_{1} \ldots a_{M-1}}^{A i_{0} i_{1} \ldots i_{M-k}-1} / B+(-1)^{k} \bar{L}_{A}^{a_{0} \ldots a_{M}} \alpha_{B a_{0} \ldots a_{M}-1, a_{M}}^{A i_{0} i_{1} \ldots i_{M}-k-1} / B+ \\
& +(-1)^{k+1} \sum_{j=0}^{k+1} L_{A}^{a_{0} a_{1} \ldots a_{M-k+j-1}} \alpha_{B a_{0} a_{1} \ldots a_{M-k+j-1}}^{A i_{0} i_{1} \ldots i_{M-k+1}} / B
\end{aligned}
$$

The fourth expression can be absorbed in the first by extending the summation in the latter from $j=k-1$ to $j=k$. In the second and third expressions we replace the summation over $j$ by one over $j-1$ and include a $j=0$ term (which is zero by (2.3)). From the sixth expression we extract the term $j=k+1$ and combine it with the fifth expression. Finally we group the remaining terms in the sixth expression with those in the third to find that the left hand side of (2.9) reads

$$
\begin{aligned}
& \sum_{j=0}^{k}(-1)^{j}\left\{\sum_{p=0}^{k-j}(-1)^{p} \bar{L}_{A}^{a_{0} \ldots a_{M}-p}, a_{M-p} \cdots a_{M-k+j}+(-1)^{k-j+1} \bar{L}_{A}^{a_{0} \ldots a_{M}-k+j-1}\right\} \times \\
& \times\left(\alpha_{B a_{0} a_{1} \ldots a_{M-k+j-1}}^{A i_{0} i_{1} \ldots i_{M-k-1}}-\alpha_{B a_{0} \ldots a_{M-k+j-2}, a_{M-k+j-1}}^{A i_{0} i_{1} \ldots i_{M-k-1}}\right) / B+ \\
& +(-1)^{k+1} \bar{L}_{A}^{a_{0} \ldots a_{M}}\left(\alpha_{B a_{0} \ldots a_{M}}^{A i_{0} \ldots a_{M-k-1}}-\alpha_{B a_{0} a_{1} \ldots a_{M}-1, a_{M}}^{A i_{0} i_{1} \ldots i_{M-k}}\right) / B .
\end{aligned}
$$

By virtue of (2.3) this is the right hand side of (2.9), which establishes the lemma.

With the aid of this lemma we are now in a position to prove

THEOREM 1. If under (1.1) $\rho^{A}$ and L transform according to (1.2) and (1.5) respectively then

$$
B E_{A}(L)=C_{A}^{B} \bar{E}_{B}(\bar{L})
$$

Proof. In (2.5) we set $k=M-1$ to find

$$
\begin{aligned}
& \sum_{p=0}^{M-2}(-1)^{p} L_{B}^{i_{0} \ldots i_{M}-r_{, i_{M}-p} \ldots i_{2}}+(-1)^{M-1} L_{\mathrm{B}}^{i_{0} i_{1}}= \\
& \quad=\sum_{j=0}^{M-2}(-1)^{j}\left\{\sum_{p=0}^{M-j-2}(-1)^{p} \bar{L}_{A}^{a_{0} \ldots a_{M}-p_{, a_{M}-p} \ldots a_{j+2}}+\right. \\
& \left.\quad+(-1)^{M-j-1} \bar{L}_{A}^{a_{0} \ldots a_{j+1}}\right\} \alpha_{B a_{0} \ldots a_{j}}^{A i_{0}} B_{a_{j+1}}^{i_{1}} / B+ \\
& \quad+(-1)^{M-1} \bar{L}_{A}^{a_{0} \ldots a_{M}} \alpha_{B a_{0} \ldots a_{M-1}}^{A i_{0}} B_{a_{M}}^{i_{1}} / B
\end{aligned}
$$


By differentiating the latter with respect to $x^{i_{1}}$, multiplying by $(-1)^{-M}$ and recalling (1.4) we see that

$$
\begin{aligned}
& E_{B}(L)=\sum_{j=0}^{M-1}(-1)^{j} \sum_{p=0}^{M-j-1}(-1)^{p-M} \bar{L}_{A}^{a_{0} \ldots a_{M-p}}{ }_{. a_{M-p} \ldots a_{j+1}} \propto_{B a_{0} \ldots a_{j}}^{A i_{0}} / B+ \\
& +\sum_{j=0}^{M-2}(-1)^{j}\left\{\sum_{p=0}^{M-k-2}(-1)^{p-M} \bar{L}_{A}^{a_{0} \ldots a_{M-n}}, a_{M}-p \ldots a_{j+2}+(-1)^{-j-1} \bar{L}_{A}^{a_{0} \ldots a_{j+1}}\right\} \times \\
& \times \alpha_{B a_{0} \ldots a_{j}, a_{j+1}}^{A i_{0}} / B-\bar{L}_{A}^{a_{0} \ldots a_{M}} \alpha_{B a_{0} \ldots a_{M-1}, a_{M}}^{A \hat{i}_{0}} / B+ \\
& +\sum_{j=0}^{M} \bar{L}_{A}^{a_{0} \ldots a_{j}} \alpha_{B a_{0} \ldots a_{j}}^{A i_{0}} / B \text {. }
\end{aligned}
$$

By virtue of (2.3) only the $j=0$ terms in the first and final expresssions survive on the right hand side - all others cancel. We thus find

$$
E_{B}(L)=\left\{\sum_{p=0}^{M-1}(-1)^{p-M} \bar{L}_{A}^{a_{0} \ldots a_{M-p}{ }_{, a_{M}-}{ }^{2} a_{1}}+\bar{L}_{A}^{a_{0}}\right\} \alpha_{B a_{0}}^{A i_{0}} / B,
$$

which, in view of (2.4), is (2.10).

From Theorem 1 we see immediately that if $\rho^{A}$ are the components of a relative tensor of covariant valency $r$, contravariant valency $s$ and weight $w$, then $E_{A}(L)$ are the components of a relative tensor of covariant valency $s$, contravariant valency $r$ and weight $(1-w)$.

We remark that Theorem 1 can be extended to quantities $\rho^{A}$ which transform according to

$$
\bar{\rho}^{A}=C_{B}^{A} \rho^{B}+\theta^{A}
$$

where the $C_{B}^{A}$ and $\theta^{A}$ are functions of $\bar{x}^{a}$ and are completely determined by the transformation (1.1). [A typical example of this would be the symmetric affine connection $\Gamma_{i j}^{h}$ which transforms according to

$$
\left.\bar{\Gamma}_{b c}^{a}=B_{b}^{m} B_{c}^{r} A_{h}^{a} \Gamma_{m}{ }^{h} r+A_{h}^{a} B_{b c}{ }^{h} \cdot\right]
$$

In fact we can prove the

THEOREM. If under (1.1) $L$ is a scalar density and $\rho^{A}$ transform according to (2.11) then

$$
B E_{A}(L)=C_{A}^{B} \widetilde{E}_{B}(\bar{L}) .
$$

However, we will not consider this case in any further detail, but will return to (1.2). If we multiply (2.10) by $\rho^{A}$ and note (1.2) we have

THEOREM 2. If under (1.1) $\rho^{A}$ and L transform according to (1.2) and (1.5) respectively then $\rho^{A} E_{A}$ is a scalar density. 
We shall now give a direct proof of the frequently asserted

THEOREM 3. A divergence satisfies the Euler-Lagrange equations identically ${ }^{4}$, i.e. if a Lagrangian $L$ is of the form

where

$$
\underset{(D)}{L}=S_{, i}^{i}
$$

$$
S^{i}=S^{i}\left(\rho^{A} ; \rho^{A}{ }_{i_{1}} ; \ldots ; \rho^{A}{ }_{i_{1} \ldots i_{M-1}-1} ; \lambda^{\alpha} ; \lambda^{\alpha}{ }_{i_{1}} ; \ldots ; \lambda^{\alpha}{ }_{i_{1} \ldots i_{p}}\right),
$$

and $p$ is any positive integer

then

$$
E_{A}(L) \equiv 0
$$

Proof. It is clear that ${ }^{5}$

$$
\text { (2.12) } \underset{(\boldsymbol{D})}{L}=\sum_{r=0}^{M-1} S^{i ;}{ }_{B}{ }^{j} j_{1} \ldots j_{r} \rho^{B}{ }_{, j_{0} j_{1} \ldots j_{r} i}+\sum_{r=0}^{p} S^{i ; j_{0} j_{1} \ldots j_{r} \lambda^{\alpha}{ }_{, j_{0} j_{1} \ldots j_{r} i}}
$$

where

and

$$
S^{i}{ }_{B}{ }^{j o j_{1} \ldots j_{r}}=\partial S^{i} / \partial \rho^{B}{ }_{, j o j_{1} \ldots j_{r}}
$$

$$
S^{i ;}{ }_{a}^{j_{0} j_{1} \ldots j_{r}}=\partial S^{i} / \partial \lambda^{\alpha}, j_{0} j_{1} \ldots j_{r} .
$$

For $1 \leqq k \leqq M-1$ it is easily seen, from (2.12), that

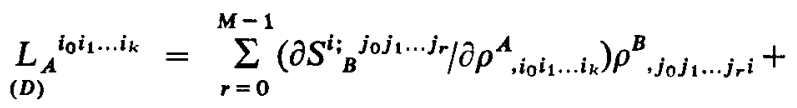

$$
\begin{aligned}
& +\sum_{r=0}^{p}\left(\partial S_{\alpha}^{i ; j_{0} j_{1} \ldots j_{r}} / \partial \rho^{A}{ }_{, i_{0} i_{1} \ldots i_{k}}\right) \lambda^{\alpha},{ }_{j_{0} j_{1} \ldots j_{r} i}+ \\
& \left.+S_{A}^{\left[i_{k} ;\right.}\left\|i_{0}\right\| i_{1} \ldots i_{k-1}\right],
\end{aligned}
$$

where the square bracket denotes complete symmetrisation over $i_{1} \ldots i_{k}$ (since $i_{0}$ is excluded from this symmetrisation process we place it in braces). However, for $1 \leqq k \leqq M-1$, it is easily seen that

$$
\begin{aligned}
& S_{A}^{i} i_{i} i_{1} \ldots i_{k}=\sum_{r=0}^{M-1}\left(\partial S_{A}^{i i_{i} i_{1} \ldots i_{k}} / \partial \rho_{, j_{0} j_{1} \ldots j_{r}}^{B}\right) \rho_{, j_{0} j_{1} \ldots j_{r} i}^{B}+ \\
&+\sum_{r=0}^{p}\left(\partial S_{A}^{i i_{i} i_{1} \ldots i_{k}} / \partial \lambda_{, j_{0} j_{1} \ldots j_{r}}^{\alpha}\right) \lambda_{, j_{0} j_{1} \ldots j_{r} i}^{\alpha}
\end{aligned}
$$

4 This result is not at variance with [1] p. 121.

5 Summation over $\alpha$ from 1 to $q$. 
which, when taken together with (2.13), yields

$$
{ }_{(D)}^{L_{A}^{i_{0} i_{1} \ldots i_{k}}}=S_{A}^{i_{i} i_{0} i_{1} \ldots i_{k}}{ }_{, i}+S_{A}^{\left[i_{k} i\left\|i_{0}\right\| i_{1} \ldots i_{k}-1\right]} .
$$

From the latter we see that for $1 \leqq k \leqq M-1$

$$
{ }_{(D)}^{L_{A}^{i_{0} i_{1} \ldots i_{k}}{ }^{, i_{0} i_{1} \ldots i_{k}}}=S_{A}^{i_{k+1} ; i_{0} i_{1} \ldots i_{k}}{ }_{, i_{0} i_{1} \ldots i_{k+1}}+S_{A}^{i_{k} ; i_{0} i_{1} \ldots i_{k}-1}{ }_{, i_{0} i_{1} \ldots i_{k}}
$$

from which we conclude that

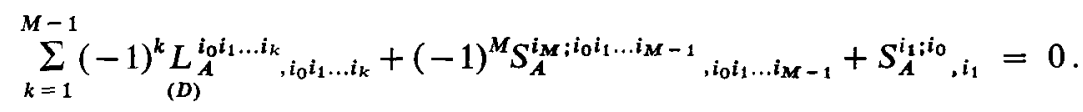

In view of (2.12) the last two terms on the left hand side are respectively the $k=M$ and $k=0$ terms of the first expression, so that

$$
E_{A}(L) \equiv 0
$$

\section{Degenerate Lagrange densities}

For the reasons indicated in section 1 we now wish to investigate the consequences of adopting the quantity

$$
\mathscr{L}=\rho^{A} E_{A}(L)
$$

as a Lagrangian. If it is assumed that the $\rho^{\boldsymbol{A}}$ transform according to (1.2) then Theorem 2 assures us that $\mathscr{L}$ is a scalar density - one of the requirements usually made of a Lagrangian. Furthermore, if $L$ is of the type (1.3) then in general $E_{A}(L)$ will involve derivatives of $\rho^{A}$ up to order $2 M$ and derivatives of $\lambda^{\alpha}$ up to order $M+Q$, in which case

$$
\mathscr{L}=\mathscr{L}\left(\rho^{A} ; \rho^{A}{ }_{, i_{1}} ; \ldots ; \rho^{A}{ }_{i_{1} i_{2} \ldots i_{2 M}} ; \lambda^{\alpha} ; \lambda^{\alpha}{ }_{, i_{1}} ; \ldots ; \lambda^{\alpha}{ }_{i_{1} i_{2}, \ldots i_{M}+Q}\right) .
$$

This in turn suggests that the associated Euler-Lagrange expression $E_{A}(\mathscr{L})$ [i.e. (1.4) with $L$ replaced by $\mathscr{L}$ and $M$ replaced by $2 M]$ will be of order $4 M$ in $\rho^{A}$. In fact this is not the case as is shown by

THEOREM 4. If

$$
L=L\left(\rho^{A} ; \rho^{A}{ }_{, i_{1}} ; \ldots ; \rho^{A}{ }_{i_{1} i_{2} \ldots i_{M}} ; \lambda^{\alpha} ; \lambda^{\alpha}{ }_{, i_{1}} ; \ldots ; \lambda^{\alpha}{ }_{, i_{1} \ldots i_{Q}}\right)
$$

and

$$
\mathscr{L}=\rho^{A} E_{A}(L)
$$

then $E_{A}(\mathscr{L})$ is at most of order $2 M$ in $\rho^{A}$ i.e. $\mathscr{L}$ is a degenerate Lagrange density. 
Proof. For any positive integer $r, 2 \leqq r \leqq M$ consider the quantity

where

$$
S^{i_{r-j}, i_{r-j}}
$$

$$
S^{i_{r-j}}=(-1)^{r-j} \rho_{,{ }_{i} i_{r-1} \ldots i_{r-j+1}}^{A} L_{A}^{i_{0} i_{1} \ldots i_{r}}{ }_{, i_{0} i_{1} \ldots i_{r-j-1}}
$$

and $1 \leqq j \leqq r-1$. Clearly we have

$$
\begin{aligned}
S^{i_{r}-j}{ }_{, i_{r}-j}= & (-1)^{r-j} \rho^{A}{ }_{, i_{r} \ldots i_{r}-j} L_{A}^{i_{0} i_{1} \ldots i_{r}}{ }_{, i_{0} i_{1} \ldots i_{r-j-1}}+ \\
& -(-1)^{r-j+1} \rho^{A}{ }_{, i_{r} \ldots i_{r-j+1}} L_{A}^{i_{i} i_{1} \ldots i_{r}}{ }_{, i_{0} \ldots i_{r-j}},
\end{aligned}
$$

from which we conclude that

$$
\sum_{j=1}^{r-1} S^{i_{r-}{ }_{, i_{r-j}}}=-\rho^{A}{ }_{,{ }_{i} i_{1} \ldots i_{r}} L_{A}^{i_{0} i_{1} \ldots i_{r}}-(-1)^{r} \rho^{A}{ }_{i_{r}} L_{A}^{i_{0} \ldots i_{r}}{ }_{, i_{0} \ldots i_{r-1}} .
$$

In view of the fact that the last term on the right hand side can be expressed in the form

$$
(-1)^{r+1}\left\{\left(\rho^{A} L_{A}^{i_{0} \ldots i_{r}, i_{0} \ldots i_{r-1}}\right)_{i_{r}}-\rho^{A} L_{A}^{i_{0} \ldots i_{r}, i_{0} \ldots i_{r}}\right\},
$$

we find that for $2 \leqq r \leqq M$

$$
\begin{aligned}
(-1)^{r} \rho^{A} L_{A}^{i_{0} \ldots i_{r}}{ }_{, i_{0} \ldots i_{r}}= & \rho^{A}{ }_{, i_{0} \ldots i_{r}} L_{A}^{i_{0} \ldots i_{r}}+\sum_{j=1}^{r-1} S^{i_{r-j}{ }_{, i_{r-j}}} \\
& +(-1)^{r}\left(\rho^{A} L_{A}^{i_{0} \ldots i_{r}}{ }_{, i_{0} \ldots i_{r-1}}\right)_{i_{r}} .
\end{aligned}
$$

Consequently we find

$$
\sum_{r=0}^{M}(-1)^{r} \rho^{A} L_{A}^{i_{0} \ldots i_{r}}{ }_{, i_{0} \ldots i_{r}}=\sum_{r=0}^{M} \rho^{A}{ }_{, i_{0} \ldots i_{r}} L_{A}^{i_{0} \ldots i_{r}}+\sum_{r=2}^{M} \sum_{j=1}^{r-1} S^{i_{r-j}{ }_{, i_{r-j}}}+
$$

$$
+\sum_{r=1}^{M}(-1)^{r}\left(\rho^{A} L_{A}^{i_{0} \ldots i_{r}, i_{0} \ldots i_{r-1}}\right\}_{, i_{r}}
$$

which, in view of (3.1), is of the form

$$
\mathscr{L}=\mathscr{L}_{1}+T^{i}{ }_{i}
$$

where

$$
\mathscr{L}_{1}=\sum_{r=0}^{M} \rho^{A}{ }_{i_{0} \ldots i_{r}} L_{A}^{i_{0} \ldots i_{r}}
$$

and 


$$
T^{i}=\sum_{r=2}^{M} \sum_{j=1}^{r-1} S^{i_{r-j} j} \delta_{i_{r-j}}^{i}+\sum_{r=1}^{M}(-1)^{r} \rho^{A} L_{A}^{i_{0} \ldots i_{r}}, i_{0} \ldots i_{r-1} \delta_{i_{r}}^{i} .
$$

However, by virtue of the fact that $T^{i}{ }_{i}$ is a divergence, Theorem 3 assures us that it will not contribute to $E_{A}(\mathscr{L})$. Therefore we find from (3.3) that

$$
E_{A}(\mathscr{L})=E_{A}\left(\mathscr{L}_{1}\right)
$$

Furthermore it is clear from (3.4) that

$$
\mathscr{L}_{1}=\mathscr{L}_{1}\left(\rho^{A} ; \rho^{A}{ }_{, i_{1}} ; \ldots ; \rho^{A}{ }_{, i_{1} \ldots i_{M}} ; \lambda^{\alpha} ; \lambda^{\alpha}{ }_{, i_{1}} ; \ldots ; \lambda^{\alpha}{ }_{, i_{1} \ldots i_{Q}}\right\},
$$

so that $E_{A}(\mathscr{L})$ is at most of order $2 M$ in $\rho^{A}$. This establishes the theorem.

We have shown that if $\mathscr{L}$, given by (3.1), is used as a Lagrangian then the corresponding Euler-Lagrange equations are also obtained from $\mathscr{L}_{1}$, given by (3.4). We also know that $\mathscr{L}$ is a scalar density. It is obviously of interest to establish the tensorial character of $\mathscr{L}_{1}$. This can easily be accomplished as follows. Under (1.1) we have

$$
\begin{aligned}
\sum_{r=0}^{M} \rho_{, i_{0} \ldots i_{r}}^{B} L_{B}^{i_{0} \ldots i_{r}} & =\sum_{r=0}^{M} \rho_{, i_{0} \ldots i_{r}}^{B} \sum_{j=0}^{M} L_{A}^{a_{0} \ldots a_{j}} \alpha_{B a_{0} \ldots a_{j}}^{A i_{0} \ldots i_{r}} / B \\
& =\sum_{j=0}^{M}\left\{\sum_{r=0}^{M} \rho_{, i_{0} \ldots i_{r}}^{B} \alpha_{B a_{0} \ldots a_{j}}^{A i_{0} \ldots a_{r}}\right\} L_{A}^{a_{0} \ldots a_{j}} / B .
\end{aligned}
$$

By (2.1) and 2.3) the quantity in brackets on the right hand side is $\bar{\rho}_{, a_{0} \ldots a_{j}}$, which establishes

THEOREM 5. The Euler-Lagrange expressions obtained from

$$
\mathscr{L}=\rho^{A} E_{A}(L)
$$

and

$$
\mathscr{L}_{1}=\sum_{r=0}^{M} \rho_{, i_{0} \ldots i_{r}}^{A} L_{A}^{i_{0} \ldots i_{r}}
$$

are identical, and, furthermore, both $\mathscr{L}$ and $\mathscr{L}_{1}$ are scalar densities.

We are now in a position to give a partial answer to the third question posed in section 1 . From Theorem 5 we see that if $\mathscr{L}_{1}$ is proportional to $L$ i.e. if

$$
\mathscr{L}_{1}=k L
$$

where $k$ is a non-zero constant then

$$
E_{A}(\mathscr{L})=k E_{A}(L)
$$

By virtue of the definition of $\mathscr{L}_{1}$ we thus have 
THEOREM 6. If $L$ is homogeneous of degree $k(\neq 0)$ in the variables $\left(\rho^{A} ; \rho^{A}{ }_{i_{1}} ; \ldots ; \rho^{A}{ }_{, i_{1} \ldots i_{M}}\right)$, i.e. if

$$
\sum_{r=0}^{M} \rho_{. i_{0} i_{1} \ldots i_{r}}^{A} L_{A}^{i_{0} i_{1} \ldots i_{r}}=k L
$$

then the Lagrange density $\rho^{A} E_{A}(L)$ will also have as its Euler-Lagrange equations precisely

$$
E_{A}(L)=0 .
$$

\section{References}

[1] D. G. B. Edelen, 'The null set of the Euler-Lagrange operator', Arch. Rational Mech. Anal. 11 (1962), 117-121.

[2] D. Lovelock, 'The uniqueness of the Einstein field equations in a four dimensional space', Arch. Rational Mech. Anal. 33, (1969), 54-70.

[3] D. Lovelock, 'Divergence-free tensorial concomitants', Aequationes Mathematicae 4 (1970), 127-138.

[4] D. Lovelock, 'Degenerate Lagrange densities involving geometric objects', Arch. Rational Mech. Anal. 36 (1970), 292-304.

[5] H. Rund, 'Variational problems involving combined tensor fields', Abh. Math. Sem. Univ. Hamburg 29 (1966), 243-262.

Department of Applied Mathematics

University of Waterloo

Waterloo, Ontario, Canada 